\title{
A LINEARIZED MODEL OF QUANTUM TRANSPORT IN THE ASYMPTOTIC REGIME OF QUANTUM WELLS
}

\author{
${ }^{1}$ A. Mantile \\ ${ }^{1}$ Laboratoire de Mathématiques, Université de Reims - FR3399 CNRS, Moulin de la \\ Housse BP 1039, 51687 Reims, France \\ andrea.mantile@univ-reims.fr
}

PACS 03.65.Xp, 02.30.Jr, 03.65.Sq
DOI 10.17586/2220-8054-2015-6-1-100-112

The effects of the local accumulation of charges in resonant tunnelling heterostructures have been described using 1D Shrödinger-Poisson Hamiltonians in the asymptotic regime of quantum wells. Taking into account the features of the underling physical system, the corresponding linearized model is naturally related to the adiabatic evolution of shape resonances on a time scale which is exponentially large w.r.t. the asymptotic parameter $h$. A possible strategy to investigate this problem consists of using a complex dilation to identify the resonances with the eigenvalues of a deformed operator. Then, the adiabatic evolution problem for a sheet-density of charges can be reformulated using the deformed dynamical system which, under suitable initial conditions, is expected to evolve following the instantaneous resonant states.

After recalling the main technical difficulties related to this approach, we introduce a modified model where $h$-dependent artificial interface conditions, occurring at the boundary of the interaction region, allow one to obtain adiabatic approximations for the relevant resonant states, while producing a small perturbation of the dynamics on the scale $h^{N_{0}}$. According to these results, we finally suggest an alternative formulation of the adiabatic problem. An a posteriori justification of our method is obtained by considering an explicitlysolvable case.

Keywords: Schrödinger-Poisson equation, adiabatic evolution of resonances.

Received: 25 January 2015

\section{Introduction}

We consider the axial transport through resonant tunneling structures like highly doped p-n semiconductor heterojunctions (Esaki diodes), multiple barriers or quantum well diodes. In such systems, the conduction band edge-profile is described by a multiple-barrier potential (see e.g. in [7]). Due to the quantum tunneling, the charge carriers, at the resonant levels, interact with metastable states and, depending on the potential geometry, a local accumulation of charges may result. The corresponding repulsive effect, which can strongly modify the transport properties, have been described in the mean-field approximation by one-particle quantum Hamiltonians of Hartree-type with Poisson nonlinearity ( [13]). In this framework, the barriers depth, fixing the time scale for the dispersion of the metastable states, can be rather large compared to the size of the wave packets. An unitarily equivalent description of the model then consists of replacing the kinetic part of the Hamiltonian with the 'semiclassical' 1D Laplacian, $-h^{2} \Delta$, while the potential is the superposition of a barrier, supported on a bounded interval $[a, b]$, and multiple potential wells with support of size $h$ inside $(a, b)$. The parameter $h$ now corresponds to a rescaled Fermi length (see for instance [6]) fixing the quantum scale of the system and, coherently with the features of the physical 
model, is assumed to be small. The resulting transport model is described by a double-scale Schrödinger-Poisson Hamiltonian:

$$
H_{N L}^{h}=-h^{2} \Delta+\mathcal{V}^{h}+V_{N L}^{h}
$$

where the linear part of the potential is defined according to:

$$
\mathcal{V}^{h}=V+W^{h}, \quad \operatorname{supp} V=[a, b],
$$

and

$$
W^{h}=-\sum_{n=1}^{N} w_{n}\left(\left(x-x_{n}\right) 1 / h\right), \quad w_{n} \in \mathcal{C}^{0}\left(\mathbb{R}, \mathbb{R}_{+}\right), \quad \operatorname{supp} w_{n}=[-d, d],
$$

for some $d>0$. For a suitable choice of $V$, this fixes a semiclassical island with quantum wells supported around the collection of points $x_{n} \in(a, b), n=1, \ldots, N$. The nonlinear potential term, $V_{N L}^{h}$, solves a Poisson equation with a source given by the charge density of the system. The corresponding evolution problem is as follows:

$$
\left\{\begin{array}{l}
i \partial_{t} u^{h}(\cdot, t, k)=\left(-h^{2} \Delta+\mathcal{V}^{h}+V_{N L}^{h}\right) u^{h}(\cdot, t, k), \\
\left(-h^{2} \Delta+\mathcal{V}^{h}+\left.V_{N L}^{h}\right|_{t=0}-k^{2}\right) u^{h}(\cdot, 0, k)=0 \\
-\Delta V_{N L}^{h}=d^{h} \\
d^{h}(x)=\int \frac{d k}{2 \pi h} g(k)\left|u^{h}(x, t, k)\right|^{2} .
\end{array}\right.
$$

Here, $u^{h}(\cdot, t, k)$ denotes the nonlinear evolution of a generalized eigenfunction $u^{h}(\cdot, 0, k)$ related to the Hamiltonian at $t=0$. The quantum state corresponding to this picture is described by a density matrix $\rho^{h}$ defined by the momenta distribution $g(k)$, and whose kernel evolves in time according to

$$
\rho_{t}^{h}(x, y)=\int \frac{d k}{2 \pi h} g(k) u^{h}(x, t, k) \bar{u}^{h}(y, t, k) .
$$

When $g(k)$ selects incoming waves (i.e.: $\operatorname{supp} g \subset \mathbb{R}_{+}$), the system is nourished by charges from the left, providing a non-equilibrium condition where, depending on the potential geometry, the particles may accumulate inside the wells. These systems - characterized by a very rich nonlinear characteristics, such as hysteresis phenomena and steadily oscillating currents - have attracted increasing interest, both for the challenging mathematical problems associated with them as well as the potential application perspectives.

Resonant energies, produced by the potential $\mathcal{V}^{h}$, naturally arise in this class of models and play a central role in the description of quantum tunneling. The incoming electrons, at resonant levels below $\sup _{(a, b)} V$, interact with resonant states which evolve in time according to a quasi-stationary dynamics. In particular, their $L^{2}$-mass remains concentrated in the vicinity of the wells support for a range of time exponentially large w.r.t. $h$. Depending on the position of the wells, this possibly induces a local charging process; then, the nonlinear coupling in (4) generates a positive response (depending upon the charge in the wells) which modifies the potential profile and reduces the tunneling rate.

This dynamics was considered in the works of G. Jona-Lasionio, C. Presilla and J. Sjöstrand ( [13], [17], [18]), within a simplified framework where the Poisson potential is replaced by an affine function multiplied by a nonlinear charge density. Using slowly varying potential assumptions, WKB expansions and a one-mode approximation for the time evolution of the quantum state, the authors discuss the behavior of the sheet density 
related to the accumulation of electrons in a single well determined by a flat double-barrier potential. Rephrasing the result of their work in our scaling, they show that the relevant time scale of the problem is on the order of $e^{\tau / h}$, corresponding to the imaginary part of the lowest resonance, and provide with an explicit equation for the evolution of the local charge density in the limit $h \rightarrow 0$ (eq. 9.7 in [17]).

It is worthwhile to note that these calculations have been shown to be relevant only in some specific cases. This concerns, in particular, the adiabatic approximation for the nonlinear evolution of generalized eigenfunctions, which appears to be an essential point of the analysis: the lack of an error bound in the adiabatic formulas for resonant states prevents the control of the possible remainder terms in the asymptotic limit. Moreover, the role played by the device's geometry in the emergence of nonlinear effects remains an open problem. This was pointed out by F.Nier, Y.Patel and V.Bonaillie-Nöel, in a series of works devoted to the steady state problem related to (4) under far-from-equilibrium assumptions. In [4]- [5], an accurate microlocal analysis of the tunneling effect as $h \rightarrow 0$ determines the limit occupation number of resonant states. This analysis leads to a simplified equation for the Poisson problem, where the limit charge density is described by a superposition of delta-shaped distributions centered in the points $x_{n}$ (see the definition (3)).

The relevance of the adiabatic approximation, for the Schrödinger-Poisson equation in the regime of quantum wells, suggests that we consider as a preliminary step, a 'linearized' problem where the nonlinear term of $H_{N L}^{h}$ is replaced by a potential supported on $(a, b)$ and adiabatically dependent on time; namely we introduce the time dependent family of Schrödinger operators:

$$
H_{0}^{h}(t)=-h^{2} \Delta+\mathcal{V}^{h}(t),
$$

where $\mathcal{V}^{h}(t)=V+W^{h}(t)$ fulfills the particular scaling (2)-(3) for all $t$. The adiabatic evolution of an observable $\chi$ (corresponding to a local charge) is described by the variable $A_{0}^{h}(t)$ solving the equation:

$$
\left\{\begin{array}{l}
A_{0}^{h}(t)=\operatorname{Tr}\left[\chi \rho_{0}^{h}(t)\right] \\
\rho_{0}^{h}(t)=\int \frac{d k}{2 \pi h} g(k)\left|u^{h}(k, \cdot, t)\right\rangle\left\langle u^{h}(k, \cdot, t)\right| \\
i \varepsilon \partial_{t} u^{h}(k, \cdot, t)=H_{0}^{h}(t) u^{h}(k, \cdot, t) \\
\left(H_{0}^{h}(t=0)-k^{2}\right) u^{h}(k, \cdot, 0)=0 .
\end{array}\right.
$$

In this framework, the relevant resonant energies for the transport problem are determined by the resonances of $H_{0}^{h}$ with the real part embedded in $\left(0, \inf _{[a, b]} V\right)$; these are usually referred to as shape resonances. In the asymptotic regime of quantum wells $(h \rightarrow 0)$, it is known that the number of shape resonances is uniformly bounded w.r.t. $h$, while their imaginary part is on the order of $e^{\tau / h}$ for a suitable constant $\tau>0$ depending on the potential geometry (for this point we refer to the analysis developed in [10]- [11]. Following [20], this quantity fixes the time scale for the dispersion of the corresponding resonant states. Hence, the effect of the accumulation of charges inside the wells can be investigated by choosing the adiabatic parameter $\varepsilon$, in $(7)$, of size $e^{-\tau / h}$.

\subsection{Detecting quantum resonances: the exterior complex dilation}

The small- $h$ analysis of the linearized problem (7), involves the following task: clarify, in an energy interval close to the shape resonances, the relation between the evolution of generalized eigenfunctions and of the resonant states in the adiabatic limit. Let us recall that 
the resonances of a Schrödinger operator correspond (modulo a restriction to a dense subset of dilation-analytic functions) to the poles of the meromorphic extension of its resolvent to the second Riemann sheet (i.e.: $\operatorname{Im} \sqrt{z}<0$ ). These are detected through the complex deformation method (see [12] for details), which can be adapted to our framework by using the exterior complex scaling: $x \rightarrow x e^{\theta 1_{\mathbb{R} \backslash(a, b)}(x)}$. When $\theta \in \mathbb{R}$, the related deformation is an unitary operator acting on $L^{2}(\mathbb{R})$ according to

$$
U_{\theta} u(x)= \begin{cases}e^{\theta / 2} u\left(e^{\theta}(x-b)-b\right), & x>b, \\ u(x), & (a, b), \\ e^{\theta / 2} u\left(e^{\theta}(x-a)-a\right), & x<a .\end{cases}
$$

For the Hamiltonian:

$$
Q=-\Delta+\mathcal{V}, \quad \text { with: } \mathcal{V} \in L^{\infty}(\mathbb{R}) \text { and supp } \mathcal{V}=[a, b]
$$

the corresponding deformed operator, next denoted with: $Q(\theta)=U_{\theta} Q U_{\theta}^{-1}$, is explicitly written as (see e.g. in [8]):

$$
Q(\theta)=-e^{-2 \theta 1_{\mathbb{R} \backslash(a, b)} \Delta_{\theta}+\mathcal{V},}
$$

where $\Delta_{\theta}$ is the non-self-adjoint point perturbation of the Laplacian defined by the interface conditions:

$$
\begin{cases}e^{-\frac{\theta}{2}} u\left(b^{+}\right)=u\left(b^{-}\right), & e^{-\frac{3}{2} \theta} u^{\prime}\left(b^{+}\right)=u^{\prime}\left(b^{-}\right), \\ e^{-\frac{\theta}{2}} u\left(a^{-}\right)=u\left(a^{+}\right), & e^{-\frac{3}{2} \theta} u^{\prime}\left(a^{-}\right)=u^{\prime}\left(a^{+}\right) .\end{cases}
$$

Namely, we have the following:

$$
\Delta_{\theta}:\left\{\begin{array}{l}
D\left(\Delta_{\theta}\right)=\left\{u \in H^{2}(\mathbb{R} \backslash\{a, b\}) \mid(11) \text { holds }\right\}, \\
\left(\Delta_{\theta} u\right)(x)=-u^{\prime \prime}(x), \quad x \in \mathbb{R} \backslash\{a, b\} .
\end{array}\right.
$$

When $\operatorname{Im} \theta>0$, this deformation produces a rotation of the essential spectrum in the second Riemann sheet: $\sigma_{\text {ess }}(Q(\theta))=e^{-2 \operatorname{Im} \theta} \mathbb{R}_{+}$; in the cone spanned by $\mathbb{R}_{+}$and $\sigma_{\text {ess }}(Q(\theta))$ the resonances of $Q$ identify with the spectral points of $Q(\theta)$. This important result was first obtained by J. Aguilar, E. Baslev and J.M. Combes in [1], [3], where the case of analytic potentials w.r.t. the uniform complex dilation $x \rightarrow x e^{\theta}$ was considered (see also [12, Theorem 16.4]). For potentials which can be complex deformed only outside a compact region, the exterior complex scaling technique appeared in [19] in the singular version that we reconsider here.

Proposition 1.1. Let $Q$ be defined by (9); the resonances of $Q$ in the cone

$$
K_{\alpha}=\{\arg z \in(-2 \alpha, 0)\}, \quad \text { with } \alpha<\frac{\pi}{4},
$$

are eigenvalues of the operators $Q(\theta)$ for $\alpha \leqslant \operatorname{Im} \theta$.

As a consequence, the resonant state associated with a resonance $E_{r e s} \in K_{\alpha} \cap \sigma(Q(\theta))$ can be defined as an eigenvector of $Q(\theta)$, with $\alpha \leqslant \operatorname{Im} \theta$, and we have

$$
\left(Q(\theta)-E_{\text {res }}\right) \psi_{E_{\text {res }}}=0, \quad \text { in } L^{2}(\mathbb{R}) .
$$

It is worth remarking that the resonances in $K_{\alpha}$ do not depend on the deformation, i.e.: if $E_{\text {res }} \in K_{\alpha} \cap \sigma(Q(\theta))$ for a given $\theta$, then, $E_{\text {res }} \in K_{\alpha} \cap \sigma\left(Q\left(\theta^{\prime}\right)\right)$ for all $\theta^{\prime}>\theta$ and $\alpha \leqslant \operatorname{Im} \theta^{\prime}$. Nevertheless the solution $\psi_{E_{\text {res }}}$ of (14) possibly depends on $\theta$ in the exterior 
region, while, according to the shape of $U_{\theta}$, the cutoff $1_{(a, b)}(x) \psi_{E_{r e s}}$ (usually refferred to as the quasi-resonant state) is independent of $\theta$.

\subsection{An alternative approach to the adiabatic problem}

The identification of the resonances for $Q$ with the spectral points of the corresponding deformed operator $Q(\theta)$ suggest an alternative, and possibly more natural, framework to study the adiabatic problem (7). Let us define the observable $\chi$ according to

$$
\chi \in \mathcal{C}^{\infty}(\mathbb{R}), \quad \operatorname{supp} \chi \subset \subset(a, b) .
$$

With this choice, $\chi$ describes the charge accumulating in a region inside $(a, b)$. Since $\chi$ commutes with the deformation map $U_{\theta}$ for all $\theta$, making use of the properties of the trace operation we have:

$$
A_{0}^{h}(t)=\operatorname{Tr}\left[\chi \rho_{0}^{h}(t)\right]=\operatorname{Tr}\left[U_{\theta}^{*} \chi U_{\theta} \rho_{0}^{h}(t)\right]=\operatorname{Tr}\left[\chi U_{\theta} \rho_{0}^{h}(t) U_{\theta}^{*}\right] .
$$

Here, $U_{\theta} \rho_{0}^{h}(t) U_{\theta}^{*}$ identifies with the evolution at time $t$ of a deformed density matrix, when the dynamical system is generated by $H_{0}^{h}(t, \theta)=U_{\theta} H_{0}^{h}(t) U_{\theta}^{-1}$. This allows us to rephrase (7) in the equivalent form:

$$
\left\{\begin{array}{l}
A_{0}^{h}(t)=\operatorname{Tr}\left[\chi \rho_{0}^{h}(t, \theta)\right] \\
\rho_{0}^{h}(t, \theta)=\int \frac{d k}{2 \pi h} g(k)\left|u_{\theta}^{h}(k, \cdot, t)\right\rangle\left\langle u_{\theta}^{h}(k, \cdot, t)\right| \\
i \varepsilon \partial_{t} u_{\theta}^{h}(k, \cdot, t)=H_{0}^{h}(t, \theta) u_{\theta}^{h}(k, \cdot, t) \\
\left(H_{0}^{h}(t=0, \theta)-k^{2}\right) u_{\theta}^{h}(k, \cdot, 0)=0 .
\end{array}\right.
$$

When $g$ selects energies close to the shape resonances, the relevant information about the solution $u_{\theta}^{h}(k, \cdot, t)$ of the adiabatic problem in (17) are related to the evolution of the resonant states. To fix this point, assume that, for a suitable choice of $\mathcal{V}^{h}(t)$ in $(6)$, the linearized operator $H_{0}^{h}(t)$ has a shape resonance $E^{h}(t)$ remaining close for all time to a limit energy $\lambda^{0}$ (in a sense which will be specified later with an explicit example). According to Proposition 1.1, we have: $E^{h}(t) \in K_{\alpha} \cap \sigma\left(H_{0}^{h}(t, \theta)\right)$ for some $\pi / 4 \geqslant \alpha>0$ and $\alpha \leqslant \operatorname{Im} \theta$, and the corresponding instantaneous resonant state $\psi_{E^{h}(t)}$ solves the eigenvalue equation (14) with $Q(\theta)=H_{0}^{h}(t, \theta)$. As far as $\operatorname{supp} g=\left\{k>0, k^{2} \sim \operatorname{Re} E_{r e s}^{h}(t) \sim \lambda^{0}\right\}$, it is expected that

$$
1_{(a, b)}(x) u_{\theta}^{h}(k, x, t) \sim 1_{(a, b)}(x) \psi^{h}(t),
$$

where $\psi^{h}(t)$ is the solution of the problem

$$
\left\{\begin{array}{l}
i \varepsilon \partial_{t} \psi^{h}(t)=H_{0}^{h}(t, \theta) \psi^{h}(t), \\
\psi^{h}(0)=\psi_{E^{h}(0)} .
\end{array}\right.
$$

For $\varepsilon=e^{-\tau / h}$, the small- $h$ asymptotics of this dynamic is related to the adiabatic evolution of an eigenvector of the initial operator $H_{0}^{h}(t=0, \theta)$ and standard results in adiabatic perturbation theory (see e.g. in [16]) would suggest to identify $\psi^{h}(t)$ with the instantaneous resonant state $\psi_{E^{h}(t)}$ (multiplied by a suitable modulation factor), with an error of size $e^{-\tau / h}$. Such an adiabatic approximation and the relation (18) could be implemented in (10) to study the asymptotic behavior of $A_{0}^{h}(t)$ as $h \rightarrow 0$.

The main difficulty in this approach is due to the fact that the complex scaling does not preserve the m-accretivity of the operator, i.e.: the quadratic form associated with $H_{0}^{h}(t, \theta)$ has an imaginary part with undetermined sign (see the eq. (1.5) in [8]); as a 
consequence, the deformed dynamics may exhibit exponential growth w.r.t. $t$. Since uniformin-time estimates for the dynamical system are necessary to prove the adiabatic theorem, the lack of this condition in our case, prevents us from developing a rigorous approach to the study of (19) in the small- $h$ limit.

\section{A modified model}

A possible strategy to overcome the lack of uniform-in-time estimates occurring in the study of the dynamical system (19) consists of modifying the physical Hamiltonian $H_{0}^{h}$ according to the following:

$$
H_{\theta}^{h}:\left\{\begin{array}{l}
D\left(H_{\theta}^{h}\right)=\left\{u \in H^{2}(\mathbb{R} \backslash\{a, b\}) \mid(11) \text { holds }\right\}, \\
\left(H_{\theta}^{h} u\right)(x)=-h^{2} u^{\prime \prime}(x)+\mathcal{V}^{h}(x) u(x), \quad x \in \mathbb{R} \backslash\{a, b\},
\end{array}\right.
$$

where, as before, the potential is formed by the superposition of a barrier supported on $[a, b]$, and a collection of quantum wells $W^{h}$ defined by (3). It worthwhile to remark that, for $\theta \neq 0, H_{\theta}^{h}$ is neither self-adjoint nor symmetric and identifies with an extension of the symmetric restriction

$$
H_{0,0}^{h}=H_{0}^{h} \uparrow\left\{u \in H^{2}(\mathbb{R}) \mid u(\alpha)=u^{\prime}(\alpha)=0, \alpha=a, b\right\} .
$$

In this connection, $H_{\theta}^{h}$ is an explicitly solvable model w.r.t. $H_{0}^{h}$ and relevant quantities, as its resolvent or generalized eigenfunctions, can be expressed in terms of corresponding non-modified quantities, related to the selfadjoint operator $H_{0}^{h}$, through non-perturbative formulas. This well-known property of point perturbations (see e.g. in [2]) provides us with a usefull tool for spectral analysis and allows us to consider the pair $\left\{H_{\theta}^{h}, H_{0}^{h}\right\}$ as a scattering system. In particular, if $1_{[a, b]} \mathcal{V}^{h}>0$, it has been shown that $H_{\theta}^{h}$ has a purely continuous spectrum coinciding with $\mathbb{R}_{+}$, provided that $\theta$ is small (depending on $h$; see [14]). A detailed analysis concerned with operators of this class has been developed (in a slightly more general framework) in [8]- [15]. In what follows, we resume their main features.

\subsection{Shape resonances in the regime of quantum wells}

Let: $\mathcal{V}^{h}=V+W^{h}$, with $V$ and $W^{h}$ defined as in (2)-(3) and fufilling the following constraints:

$$
1_{[a, b]} V \geqslant c, \quad \sup \left\{\|V\|_{L^{\infty}(\mathbb{R})},\left\|W^{h}\right\|_{L^{\infty}(\mathbb{R})}\right\} \leqslant \frac{1}{c}, \quad \operatorname{supp} W^{h} \subset \subset(a, b),
$$

for some $c>0$, uniformly w.r.t. $h \in\left(0, h_{0}\right]$. It is known that this particular potential's shape prevents the accumulation of the possible eigenvalues of the corresponding 'Dirichlet operator', $H_{D}^{h}$,

$$
H_{D}^{h}=-h^{2} \partial_{x}^{2}+\mathcal{V}^{h}, \quad D\left(H_{D}^{h}\right)=H^{2}([a, b]) \cap H_{0}^{1}([a, b]),
$$

in the energy region $\left(c, \inf _{[a, b]} V\right)$ when the limit $h \rightarrow 0$ is considered (e.g. in [4]). Then, we assume $\mathcal{V}^{h}$ to verify the following spectral condition. 
Condition 2.1. There exists a real $\lambda^{0}$ and a cluster of eigenvalues $\left\{\lambda_{j}^{h}\right\}_{j=1}^{\ell} \subset \sigma\left(H_{D}^{h}\right)$ such that the conditions

$$
\begin{aligned}
& \text { i) } \quad c \leqslant \lambda^{0} \leqslant \inf _{[a, b]} V-c \leqslant\|V\|_{L^{\infty}(\mathbb{R})} \leqslant \frac{1}{c}, \\
& \text { ii) } \quad d\left(\lambda^{0}, \sigma\left(H_{D}^{h}\right) \backslash\left\{\lambda_{j}^{h}\right\}_{j=1}^{\ell}\right) \geqslant c, \\
& \text { iii) } \quad \max _{1 \leqslant j \leqslant \ell}\left|\lambda_{j}^{h}-\lambda^{0}\right| \leqslant \frac{h}{c} .
\end{aligned}
$$

hold for all $h \in\left(0, h_{0}\right]$.

Under the action of an exterior dilation $U_{i \gamma}$ with $\gamma>0$ (see the definition 8), the modified Hamiltonian $H_{\theta}^{h}$ transforms according to (e.g. in [8])

$$
H_{\theta}^{h}(i \gamma)=-h^{2} \eta(x) \Delta_{\theta+i \gamma}+\mathcal{V}^{h}, \quad \eta(x)=e^{-2 i \gamma 1_{\mathbb{R} \backslash(a, b)}(x)},
$$

where the operator's domain is obtained from (20) by replacing $\theta$ by $\theta+i \gamma$ in the interface conditions (11). In the exterior domain, the solution $\varphi_{\text {res }}$ of the eigenvalue problem corresponding to a resonance $z_{\text {res }} \in\{p \in \mathbb{C} \mid \arg p \in(-2 \gamma, 0)\}$

$$
\left(H_{\theta}^{h}(i \gamma)-z_{\text {res }}\right) \varphi_{\text {res }}=0, \quad \varphi_{\text {res }} \in L^{2}(\mathbb{R}),
$$

exhibits the exponential modes

$$
\begin{cases}\varphi_{\text {res }}(x)=c_{+} e^{i \frac{\left(z_{\text {res }}\right)^{1 / 2} e^{i \gamma}}{h}(x-b)}, & x>b, \\ \varphi_{\text {res }}(x)=c_{-} e^{i \frac{\left(z_{\text {res }}\right)^{1 / 2} e^{i \gamma}}{h}(a-x)}, & x<a .\end{cases}
$$

Let $\mathcal{P}_{z}^{h}(\theta)=\left(-h^{2} \Delta_{\theta}+\mathcal{V}^{h}\right)$

$$
D\left(\mathcal{P}_{z}^{h}(\theta)\right)=\left\{u \in H^{2}((a, b)),\left(h \partial_{x}+i z^{1 / 2} e^{-\theta}\right) u(a)=0,\left(h \partial_{x}-i z^{1 / 2} e^{-\theta}\right) u(b)=0\right\},
$$

where $(z)^{1 / 2}$ is determined according to $\arg z \in\left[-\frac{\pi}{2}, \frac{3}{2} \pi\right)$. Using (27), the resonance equation (26) is re-termed as a non-linear eigenvalue problem for the operator $\mathcal{P}_{z}^{h}(\theta)$

$$
\left(\mathcal{P}_{z_{\text {res }}}^{h}(\theta)-z_{\text {res }}\right) \varphi_{\text {res }}=0 .
$$

It is worthwhile to note that this equation does not depend on the deformation, but only on the interface conditions in (20). Following the approach of Helffer-Sjöstrand (see [11], [21]), the resonances for the full Hamiltonian $H_{\theta}^{h}(i \gamma)$ can be investigated after reducing the Grushin problem modeled from the Dirichlet operator $H_{D}^{h}$ for the boundary value operator $\mathcal{P}_{z_{\text {res }}}^{h}(\theta)$. This analysis, developed in [8], shows that, for potentials fulfilling the conditions $(24)$, the shape resonances are localized in small regions of the corresponding Dirichlet's eigenvalues $\lambda_{j}^{h}$ as $h \rightarrow 0$. In particular, let $\omega_{\delta}$ denotes the regions:

$$
\omega_{\delta}=\left\{z \in \mathbb{C}, d\left(z,\left\{\lambda_{j}^{h}\right\}_{j=1}^{\ell}\right) \leqslant \delta\right\}
$$

and $d_{A g}(x, y, \mathcal{V}, \lambda)$

$$
d_{A g}(x, y, \mathcal{V}, \lambda)=\int_{y}^{x} \sqrt{(\mathcal{V}(s)-\lambda)_{+}} d s, \quad x \geqslant y,
$$

the Agmon distance between $x$ and $y$ related to a potential $\mathcal{V}$ and an energy $\lambda \in \mathbb{R}_{+}$. 
Proposition 2.1. Let $\mathcal{V}^{h}=V+W^{h}$ be defined according to (2)-(3), (22), and assume the conditions (24) to hold. Then, for all $h \in\left(0, h_{0}\right]$ and $|\theta| \leqslant \frac{c^{2} h}{8}$, the operator $H_{\theta}^{h}$ has exactly $\ell$ resonances in $\omega_{\frac{c h}{2}},\left\{z_{j}^{h}\right\}_{j=1}^{\ell}$, possibly counted with multiciplicities. Considered as functions of $h$, after the proper labelling w.r.t. $j$, these fulfill the relations:

$$
z_{j}^{h}(\theta)-\lambda_{j}^{h}=\mathcal{O}\left(h^{-3} e^{-\frac{2 S_{0}}{h}}\right) .
$$

where $S_{0}=d_{A g}\left(\{a, b\}, \cup_{k=1}^{N}\left\{x_{k}\right\}, V, \lambda^{0}\right)$ is the Agmon distance between the asymptotic support of $W^{h}$ and the barrier's boundary. Under the additional condition

$$
\lim _{h \rightarrow 0} h^{3} e^{\frac{2 S_{0}}{h}} \min _{j \neq j^{\prime}}\left|\lambda_{j}^{h}-\lambda_{j^{\prime}}^{h}\right|=+\infty,
$$

the variations of $z_{j}^{h}(\theta)-z_{j}^{h}(0)$ is estimated by the following:

$$
\max _{j \in\{1 \ldots \ell\}}\left|z_{j}^{h}(\theta)-z_{j}^{h}(0)\right|=\mathcal{O}\left(|\theta| h^{-3} e^{-\frac{2 S_{0}}{h}}\right),
$$

for all $e^{-\frac{2 S_{0}}{h}}<|\theta|<\frac{c^{2} h}{8}$.

As a consequence of (32), the size of the imaginary part of the shape resonances is: $\left|\operatorname{Im} z_{j}^{h}(\theta)\right| \lesssim h^{-3} e^{-\frac{2 S_{0}}{h}}$. When the quantum evolution generated by $H_{\theta}^{h}$ is considered for initial states with energies close to $z_{j}^{h}(\theta)$, it is important to have a lower bound for $\operatorname{Im} z_{j}^{h}(\theta)$ as $h \rightarrow 0$. Providing such a lower bound is a standard result in semiclassical analysis; in [5][8] this problem is analyzed for Schrödinger operators depending on the scaling parameter $h$ according to the rules prescribed for $\mathcal{V}^{h}$. Under some additional spectral assumptions, it is shown that:

$$
\left|\operatorname{Im} z_{j}^{h}(\theta)\right| \gtrsim e^{-\frac{2 S_{0}}{h}}
$$

In what follows we will assume that this lower bound holds.

\subsection{The quantum evolution problem}

We next consider the time propagator generated by the operator $H_{\theta}^{h}$. In a slightly more general framework, a detailed analysis of this problem has been developed in [15], where the modified dynamics is defined through a similarity, between $H_{\theta}^{h}$ and the corresponding self-adjoint model $H_{0}^{h}$, holding in some spectral subspace under the assumption that the parameters $\theta$ and $h$ are small.

The generalized eigenfunctions of $H_{\theta}^{h}$, next denoted with $\psi_{\theta}^{h}\left(\cdot, k, \mathcal{V}^{h}\right)$, solve of the boundary value problem (we refer to [14]- [15])

$$
\left\{\begin{array}{l}
\left(-h^{2} \partial_{x}^{2}+\mathcal{V}^{h}\right) u=k^{2} u, \quad x \in \mathbb{R} \backslash\{a, b\}, k \in \mathbb{R}, \\
e^{-\frac{\theta}{2}} u\left(b^{+}\right)=u\left(b^{-}\right), \quad e^{-\frac{3}{2} \theta} u^{\prime}\left(b^{+}\right)=u^{\prime}\left(b^{-}\right), \\
e^{-\frac{\theta}{2}} u\left(a^{-}\right)=u\left(a^{+}\right), \quad e^{-\frac{3}{2} \theta} u^{\prime}\left(a^{-}\right)=u^{\prime}\left(a^{+}\right),
\end{array}\right.
$$

and fulfill the exterior conditions

$$
\begin{aligned}
& \left.\psi_{\theta}^{h}\left(x, k, \mathcal{V}^{h}\right)\right|_{\substack{x<a \\
k>0}}=e^{i \frac{k}{h} x}+R^{h}(k, \theta) e^{-i \frac{k}{h} x},\left.\quad \psi_{\theta}^{h}\left(x, k, \mathcal{V}^{h}\right)\right|_{\substack{x>b \\
k>0}}=T^{h}(k, \theta) e^{i \frac{k}{h} x}, \\
& \left.\psi_{\theta}^{h}\left(x, k, \mathcal{V}^{h}\right)\right|_{\substack{x<a \\
k<0}}=T^{h}(k, \theta) e^{i \frac{k}{h} x},\left.\quad \psi_{\theta}^{h}\left(x, k, \mathcal{V}^{h}\right)\right|_{\substack{x>b \\
k<0}}=e^{i \frac{k}{h} x}+R^{h}(k, \theta) e^{-i \frac{k}{h} x},
\end{aligned}
$$


describing an incoming wave function of momentum $k$ with reflection and transmission coefficients $R^{h}$ and $T^{h}$. In the case $\theta=0$, the generalized Fourier transform associated with $\mathcal{H}_{0}^{h}\left(\mathcal{V}^{h}\right)$ is defined by:

$$
\left(\mathcal{F}^{h} \varphi\right)(k)=\int_{\mathbb{R}} \frac{d x}{(2 \pi h)^{1 / 2}}\left(\psi_{0}^{h}\left(x, k, \mathcal{V}^{h}\right)\right)^{*} \varphi(x), \quad \varphi \in L^{2}(\mathbb{R}) .
$$

Recall that $\mathcal{F}^{h}$ is a bounded operator on $L^{2}(\mathbb{R})$ with a right inverse coinciding with the adjoint $\left(\mathcal{F}^{h}\right)^{*}$

$$
\left(\mathcal{F}^{h}\right)^{*} f(x)=\int \frac{d k}{(2 \pi h)^{1 / 2}} \psi_{0}^{h}\left(x, k, \mathcal{V}^{h}\right) f(k) .
$$

In particular, it results: $\mathcal{F}^{h}\left(\mathcal{F}^{h}\right)^{*}=\mathbb{I}$ in $L^{2}(\mathbb{R})$, while the product $\left(\mathcal{F}^{h}\right)^{*} \mathcal{F}^{h}$ defines the projector on the absolutely continuous subspace of $\mathcal{H}_{0}^{h}\left(\mathcal{V}^{h}\right)$ (cf. [22]).

We are interested in the quantum evolution for initial states residing in a spectral subspace of energies close to $\lambda^{0}$ (the eccumulation point of the resonances); let assume the interval $\left[\Lambda_{1}, \Lambda_{2}\right] \subset \mathbb{R}$ such that:

$$
c \leqslant \Lambda_{1}<\Lambda_{2} \leqslant \inf _{[a, b]} V-c, \quad \sigma\left(H_{D}^{h}\right) \cap\left[\Lambda_{1}, \Lambda_{2}\right]=\left\{\lambda_{j}^{h}\right\}_{j=1}^{\ell},
$$

uniformly w.r.t. $h \in\left(0, h_{0}\right]$. The spectral projector on $\left[\Lambda_{1}, \Lambda_{2}\right]$ associated to $H_{0}^{h}$ is next denoted with $\Pi_{\left[\Lambda_{1}, \Lambda_{2}\right]}$; this is explicitly given by:

$$
\Pi_{\left[\Lambda_{1}, \Lambda_{2}\right]} \varphi=\int_{\mathbb{R}} \frac{d k}{(2 \pi h)^{1 / 2}} 1_{\left[\Lambda_{1}, \Lambda_{2}\right]}\left(k^{2}\right) \psi_{0}^{h}\left(x, k, \mathcal{V}^{h}\right)\left(\mathcal{F}_{\mathcal{V}^{h}}^{h} \varphi\right)(k) .
$$

The similarity between $H_{\theta}^{h}$ and $H_{0}^{h}$ on the subspace $\Pi_{\left[\Lambda_{1}, \Lambda_{2}\right]} \varphi L^{2}(\mathbb{R})$ is provided by the operators $\mathcal{W}_{\theta}^{h}$ defined through the integral kernel:

$$
\mathcal{W}_{\theta}^{h}(x, y)=\int_{\mathbb{R}} \frac{d k}{2 \pi h} 1_{\left[\Lambda_{1}, \Lambda_{2}\right]}\left(k^{2}\right) \psi_{\theta}^{h}\left(x, k, \mathcal{V}^{h}\right)\left(\psi_{0}^{h}\left(x, k, \mathcal{V}^{h}\right)\right)^{*}
$$

The next proposition rephrases in our case the result presented in [15] .

Proposition 2.2. Let $\mathcal{V}^{h}=V+W^{h}$ satisfy the conditions (24) and $|\theta| \lesssim h^{N_{0}}$, with $N_{0} \geqslant 4$. If the interval $\left[\Lambda_{1}, \Lambda_{2}\right]$ verifies (41) and the lower bound (35) holds, then there exists $\eta>0$ such that: $\left\{\mathcal{W}_{\theta}^{h}, \theta \in \mathbb{C}, \mathcal{B}|\theta|<\eta h^{N_{0}}\right\}$ form an analytic family of bounded operators in $L^{2}(\mathbb{R})$ fulfilling the expansion:

$$
\mathcal{W}_{\theta}^{h}-\Pi_{\left[\Lambda_{1}, \Lambda_{2}\right]} \varphi=\mathcal{O}\left(h^{N_{0}-2}\right)
$$

in the $\mathcal{L}\left(L^{2}(\mathbb{R})\right)$ operator norm. For $|\theta|<\eta h^{N_{0}}$, the operators $\mathcal{W}_{\theta}^{h}, \mathcal{W}_{\theta}^{h} H_{0}^{h}$ and $H_{\theta}^{h} \mathcal{W}_{\theta}^{h}$ map $L^{2}(\mathbb{R})$ into $D\left(H_{\theta}^{h}\right)$, and it results

$$
H_{\theta}^{h} \mathcal{W}_{\theta}^{h}=\mathcal{W}_{\theta}^{h} H_{0}^{h}
$$

As a consequence of (44)-(45), the modified quantum evolution can be defined through the unitary propagator $e^{-i t H_{0}^{h}}$ by conjugation as far as the initial state is considered in $\Pi_{\left[\Lambda_{1}, \Lambda_{2}\right]} L^{2}(\mathbb{R})$.

Theorem 2.1. Let $h \in\left(0, h_{0}\right]$ and $|\theta| \lesssim h^{N_{0}}$, with $N_{0} \geqslant 4$. Under the assumptions of the proposition 2.2, $i H_{\theta}^{h}$ generates a strongly continuous group of bounded operators on $\Pi_{\left[\Lambda_{1}, \Lambda_{2}\right]} L^{2}(\mathbb{R})$. For a fixed $t, e^{-i t H_{\theta}^{h}}$ is analytic w.r.t. $\theta$ and the expansion:

$$
\left(e^{-i t H_{\theta}^{h}}-e^{-i t H_{0}^{h}}\right) \Pi_{\left[\Lambda_{1}, \Lambda_{2}\right]}=\tilde{\mathcal{R}}^{h}(t, \theta),
$$


holds with:

$$
\sup _{t \in \mathbb{R}}\left\|\tilde{\mathcal{R}}^{h}(t)\right\|_{\mathcal{L}\left(L^{2}(\mathbb{R})\right)}=\mathcal{O}\left(h^{N_{0}-2}\right)
$$

\subsection{Adiabatic evolution of shape resonances}

We next consider a non-autonomous Hamiltonian defined by (20) through a time dependent potential $\mathcal{V}^{h}(t)$ such that the spectral profile of $H_{\theta}^{h}(t)$ corresponds to the pitcure given in the Proposition 2.1 for any time. In particular, the potential is assigned according to the following conditions:

Condition 2.2. $\mathcal{V}^{h}(t)=V(t)+W^{h}(t)$, with $V, W^{h} \in \mathcal{C}^{K}\left(\mathbb{R}, L^{\infty}(\mathbb{R})\right)$ for $K \geqslant 2$, supp $V(t)=$ $[a, b]$ and

$$
W^{h}(t)=-\sum_{n=1}^{N} w_{n}\left(t,\left(x-x_{n}\right) 1 / h\right), \quad \operatorname{supp} w_{n}(t)=[-d, d] .
$$

We assume that for all $t$ the estimates (22) and the conditions (24) hold with $\lambda^{0} \in \mathcal{C}^{K}(\mathbb{R})$ and $c>0$ fixed.

Under these assumptions, $H_{\theta}^{h}(t)$ has a cluster of time dependent shape resonances $\left\{z_{j}^{h}(\theta, t)\right\}_{j=1}^{\ell}$ which, for $\gamma>0$ large enough, belong to $\sigma\left(H_{\theta}^{h}(i \gamma, t)\right)$, with $H_{\theta}^{h}(i \gamma, t)$ denoting the exterior complex-dilation of $H_{\theta}^{h}(t)$. Since $\sigma_{\text {ess }}\left(H_{\theta}^{h}(i \gamma)\right)=e^{-2 i \gamma} \mathbb{R}_{+}$, due to the semiclassical estimates (32) and the assumption $(i i)-(24),\left\{z_{j}^{h}(\theta, t)\right\}_{j=1}^{\ell}$ is an isolated part of the spectrum of $H_{\theta}^{h}(i \gamma, t)$. Denoting with $\Pi_{z_{j}^{h}(\theta, t)}$ the corresponding time-dependent (nonorthogonal) projetcor, the adiabatic problem for this cluster of spectral points is written as:

$$
\left\{\begin{array}{l}
i \varepsilon \partial_{t} u_{t}^{h}=H_{\theta}^{h}(i \gamma, t) u_{t}^{h} \\
u_{t=0}^{h} \in \Pi_{z_{j}^{h}(\theta, t)} L^{2}(\mathbb{R})
\end{array}\right.
$$

If the dynamical system generated by $H_{\theta}^{h}(i \gamma, t)$ allows uniform-in-time estimates, this problem can be analyzed following the standard approach of the adiabatic theorem with spectral gap condition. For a particular choice of the deformation, the modification introduced by the $\theta$-dependent interface conditions allows to obtain such a result. Indeed, the deformation $H_{\theta}^{h}(i \gamma)$ is characterized as follows (Lemma 3.1 in [8]):

Lemma 2.2. Let $\theta=i \gamma, \gamma>0$. Then: $i H_{i \gamma}^{h}(i \gamma)$ is maximal accretive.

It follows that, for each fixed $t, e^{-i s H_{i \gamma}^{h}(i \gamma, t)}$ is a semigroup of contractions; then, the regularity-in-time of the potential allows us to conclude that the non-autonomous Hamiltonian $H_{i \gamma}^{h}(i \gamma, t)$ generates a quantum dynamical system of contractions (see [8, Proposition 3.7]). In the application perspectives, we need to minimize the error produced by modification of the Hamiltonian; hence, we next assume $\gamma$ to be polynomially small w.r.t. $h$. In this framework, the resonances $z_{j}^{h}(i \gamma, t)$ are close to the essential spectrum (located in $e^{-i 2 \gamma} \mathbb{R}_{+}$) and the spectral gap condition depends on $h$. Then the estimates of $\partial_{t}^{k}\left(H_{\theta}^{h}(i \gamma, t)-z\right)$ in the region: $\left|z-z_{j}^{h}(\theta, t)\right| \sim \gamma$, behave as $\gamma^{-1}$. This entails a loss of some power for $h$ (depending on $\gamma$ ) in the adiabatic formula. Nevertheless, assuming $\varepsilon=e^{-\tau / h}$ for some $\tau>0$, it is still possible to obtain an error bound exponentially small w.r.t. $h$. Next, we recall, in the simplified case of a single shape resonance, the result of the adiabatic theorem provided with in [8, Theorem 7.1] (see also [9] for the explicit form of the modulation factor $\mu(t)$ below). 
Theorem 2.3. Let $\mathcal{V}^{h}(t)$ fulfill the Condition 2.2 with $\ell=1$, and let $E^{h}(t), \psi_{E^{h}(t)}$ respectively denote the shape resonance end the resonant state related to $H_{i h^{N}}^{h}\left(i h^{N}, t\right)$ in the neighbourhood of size $h$ of the limit energy $\lambda^{0}$. We set $\varepsilon=e^{-\tau / h}$ with $\tau>0$, and assume that

$$
\left\|\psi_{E^{h}(t)}-\psi_{E^{h}(t)}^{*}\right\|_{L^{2}(\mathbb{R})} \leqslant \varepsilon^{1-\delta}
$$

holds for any $\delta \in(0,1)$. Then, the solution $\psi^{h}(t)$ of the problem: $0 \leqslant t \leqslant T$

$$
\left\{\begin{array}{l}
i \varepsilon \partial_{t} \psi^{h}(t)=H_{i h^{N}}^{h}\left(i h^{N}, t\right) \psi^{h}(t) \\
\psi^{h}(0)=\psi_{E^{h}(0)}
\end{array}\right.
$$

fulfills the estimate:

$$
\max _{t \in[0 T]}\left\|\psi^{h}(t)-v^{h}(t)\right\|_{L^{2}(\mathbb{R})} \leqslant C_{a, b, c, \delta, T} \varepsilon^{1-\delta}\left\|\psi_{E^{h}(0)}\right\|_{L^{2}(\mathbb{R})},
$$

where the auxiliary dynamics $v^{h}(t)$ is expressed as:

$$
v^{h}(t)=\mu(t) e^{-\frac{i}{\varepsilon} \int_{0}^{t} E^{h}(s) d s} \psi_{E^{h}(t)},
$$

with

$$
\mu(t)=\exp \left(\int_{0}^{t} \frac{\left\langle\psi_{E^{h}(s)}, \partial_{s} \psi_{E^{h}(t)}\right\rangle}{\left\|\psi_{E^{h}(s)}\right\|_{L^{2}(\mathbb{R})}^{2}} d s\right)+\mathcal{O}\left(\varepsilon^{1-\delta}\right),
$$

while the positive constant $C_{a, b, c, \delta, T}$ possibly depends on the data.

\section{A conjecture for the linearized transport problem}

We finally reconsider the linearized verion of the transport problem introduced in (7). According to the result of Theorem 2.1, for initial states characterized by energies close to a cluster of resonaces allowing the lower bound (35), the distance between the propagators $\exp \left(-i t H_{i h^{N_{0}}}^{h}\right)$ and $\exp \left(-i t H_{0}^{h}\right)$ is controlled uniformely-in-time in the $L^{2}$-operator norm by $\mathcal{O}\left(h^{N_{0}-2}\right)$ for $N_{0} \geqslant 4$. In the non-autonomous case, this suggests that, under the same initial conditions, the quantum dynamical systems generated by $H_{i h^{N_{0}}}^{h}(t)$ and $H_{0}^{h}(t)$ remain close to each other. In particular, we conjecture that on the adiabatic time-scale, the result of Theorem 2.1 extends according to:

$$
\sup _{t \in[0, T]}\left\|u^{h}(t)-u_{0}^{h}(t)\right\|_{L^{2}(\mathbb{R})}=\mathcal{O}\left(h^{N_{0}-2}\right),
$$

where $u^{h}(t)$ and $u_{0}^{h}(t)$ solve the equations: $i \varepsilon \partial_{t} u^{h}(t)=H_{i h^{N_{0}}}^{h}(t) u^{h}(t)$ and $i \varepsilon \partial_{t} u_{0}^{h}(t)=$ $H_{0}^{h}(t) u_{0}^{h}(t)$ with $u_{0}^{h}(0)=u^{h}(0) \in \Pi_{\left[\Lambda_{1}, \Lambda_{2}\right]} L^{2}(\mathbb{R})$. When supp $g=\left\{k>0,\left|k^{2}-\lambda^{0}\right|=\mathcal{O}(h)\right\}$, this initial condition is almost fufilled by the modified problem

$$
\left\{\begin{array}{l}
A^{h}(t)=\operatorname{Tr}\left[\chi \rho^{h}(t)\right], \\
\rho^{h}(t)=\int \frac{d k}{2 \pi h} g(k)\left|u^{h}(k, \cdot, t)\right\rangle\left\langle u^{h}(k, \cdot, t)\right|, \\
i \varepsilon \partial_{t} u^{h}(k, \cdot, t)=H_{i h^{N_{0}}}^{h}(t) u^{h}(k, \cdot, t), \\
\left(H_{i h^{N_{0}}}^{h}(t=0)-k^{2}\right) u^{h}(k, \cdot, 0)=0,
\end{array}\right.
$$

and the solution $A_{0}^{h}(t)$ in $(7)$ is expected to be related to $A^{h}(t)$ by:

$$
A_{0}^{h}(t)=A^{h}(t)+\mathcal{O}\left(h^{N_{0}-2}\right) .
$$


Let the observable $\chi$ be defined by (15); proceeding as in (16), we have:

$$
A^{h}(t)=\operatorname{Tr}\left[\chi \rho^{h}(t)\right]=\operatorname{Tr}\left[U_{\theta}^{*} \chi U_{\theta} \rho^{h}(t)\right]=\operatorname{Tr}\left[\chi U_{\theta} \rho^{h}(t) U_{\theta}^{*}\right] .
$$

Noticing that $U_{\theta} \rho^{h}(t) U_{\theta}^{*}$ identifies with the time evolution generated by the deformed operators $H_{i h^{N_{0}}}^{h}\left(i h^{N_{0}}, t\right)=U_{\theta} H_{i h^{N_{0}}}^{h}(t) U_{\theta}^{-1}$, the problem (56) is rewritten as

$$
\left\{\begin{array}{l}
A^{h}(t)=\operatorname{Tr}\left[\chi \tilde{\rho}^{h}(t)\right], \\
\tilde{\rho}^{h}(t)=\int \frac{d k}{2 \pi h} g(k)\left|\tilde{u}^{h}(k, \cdot, t)\right\rangle\left\langle\tilde{u}^{h}(k, \cdot, t)\right|, \\
i \varepsilon \partial_{t} \tilde{u}^{h}(k, \cdot, t)=H_{i h^{N_{0}}}^{h}\left(i h^{N_{0}}, t\right) \tilde{u}^{h}(k, \cdot, t), \\
\left(H_{i h^{N_{0}}}^{h}\left(i h^{N_{0}}, t=0\right)-k^{2}\right) \tilde{u}^{h}(k, \cdot, 0)=0 .
\end{array}\right.
$$

In this equivalent formulation, the result of Theorem 2.3 applies and the evolution of resonant states related to shape resonances is properly described by adiabatic formulas of the type (52). This clarifies the idea of our approach: the modification of the physical model (9) by $h$ dependent artificial interface conditions, although introducing a small error on the solution $A_{0}^{h}(t)$ (controlled by a power of $h$ ), allows us to work in the complex deformed setting (59) where, under the condition (18), an adiabatic approximation holds for the deformed dynamics $\tilde{u}^{h}(k, \cdot, t)$.

\subsection{An explicit example and final remarks}

A rigorous justification of the method described in this work is still an open problem which mainly concerns the validity of conjecture (55), allowing one to compare the modified variable $A^{h}(t)$ and the physical one $A_{0}^{h}(t)$.

In [9], the small- $h$ behavior of the solution of (56) has been investigated for a time dependent potential formed by a flat barrier of height $V_{0}>0$ plus an attractive, timedependent delta interaction acting in $x_{0} \in(a, b)$ and preserving the quantum-well scaling. Explicitly, we consider the model:

$$
H_{i h^{N_{0}}}^{h}(t)=-h^{2} \Delta_{i h^{N_{0}}}+1_{[a, b]}(x) V_{0}+h \alpha(t) \delta_{x_{0}} .
$$

Under suitable assumptions on $\alpha(t)$ (corresponding to the Condition 2.2), these operators have a single shape resonance $E^{h}(t)$ localized close to a positive energy below $V_{0}$. In this framework, an explicit formula for the deformed dynamics $\tilde{u}^{h}(k, \cdot, t)$ and direct computations allow us to prove that the relations (18), (50) hold; then, the asymptotic behavior of $A^{h}(t)$ as $h \rightarrow 0$ can be analyzed using the result (52). In a configuration where the scattering involves only incoming from the left wave functions and the interaction point $x_{0}$ is closer to the l.h.s. of the barrier (i.e.: $\left|x_{0}-a\right|<\left|x_{0}-b\right|$ ), $A^{h}(t)$ exhibits the decomposition:

$$
A^{h}(t)=a(t)+\mathcal{J}(t)+\mathcal{O}\left(h^{N_{0}}\right),
$$

where the main term $a(t)=\mathcal{O}(1)$ solves a limit equation describing the charging process of the well, while the first error term is $\mathcal{J}(t)=\mathcal{O}\left(h^{2}\right)$ (we refer to [9, Theorem 2.1]). In particular, our limit equation is coherent with the nonlinear reduced model obtained in [13], while the interface conditions do not affect the main contributions to $A^{h}(t)$ when $N_{0}$ is large enough. Hence, this result appears as an indirect confirmation of the validity of the expansion conjectured in (55). 


\section{Acknowledgements}

We gratefully aknowledge the financial support from the CNRS (FR3399) and from the ITMO University of Saint Petersbourg.

\section{References}

[1] J. Aguilar, J.M. Combes. A class of analytic perturbations for one-body Schrödinger Hamiltonians. Comm. Math. Phys., 22, P. 269-279 (1971).

[2] S. Albeverio, F. Gesztesy, R. Høegh-Krohn, H. Holden. Solvable models in quantum mechanics, Second edition. With an appendix by Pavel Exner. AMS Chelsea Publishing, Providence, RI (2005).

[3] E. Balslev, J.M. Combes. Spectral properties of many-body Schrödinger operators with dilatationanalytic interactions. Comm. Math. Phys., 22, P. 280-294 (1971).

[4] V. Bonnaillie-Noël, F. Nier, Y. Patel. Far from equlibrium steady states of 1D-Schrödinger-Poisson systems with quantum wells I. Ann. I.H.P. An. Non Linéaire, 25, P. 937-968 (2008).

[5] V. Bonnaillie-Noël, F. Nier, Y. Patel. Far from equlibrium steady states of 1D-Schrödinger-Poisson systems with quantum wells II. J. Math. Soc. of Japan., 61, P. 65-106 (2009).

[6] V. Bonnaillie-Noël, F. Nier, Y. Patel. Computing the steady states for an asymptotic model of quantum transport in resonant heterostructures. Journal of Computational Physics, 219(2), P. 644-670 (2006).

[7] F. Capasso (Ed.). Physics of quantum electronic devices. Springer-Verlag, Berlin (1990).

[8] A. Faraj, A. Mantile, F. Nier. Adiabatic evolution of 1D shape resonances: an artificial interface conditions approach. M3AS, 21(3), P. 541-618 (2011).

[9] A. Faraj, A. Mantile, F. Nier. An explicit model for the adiabatic evolution of quantum observables driven by 1D shape resonances. J. Phys. A: Math. Theor., 43 (2010).

[10] B. Helffer. Semiclassical analysis for the Schrödinger operator and applications, volume 1336 of Lecture Notes in Mathematics. Springer-Verlag, Berlin (1988).

[11] B. Helffer, J. Sjöstrand. Résonances en limite semi-classique. Mém. Soc. Mat. France (N.S.), number 24-25, 1986.

[12] P.D. Hislop, I.M. Sigal. Introduction to spectral theory (With applications to Schrödinger operators). Volume 113 of Applied Mathematical Sciences, Springer-Verlag, New York (1996).

[13] G. Jona-Lasionio, C. Presilla, J. Sjöstrand. On the Schrödinger equation with concentrated non linearities. Ann. Physics, 240(1), P. 1-21 (1995).

[14] A. Mantile. Wave operators, similarity and dynamics for a class of Schrödinger operators with generic non-mixed interface conditions in 1D. J. Math. Phys., 54(8), P. 082104 (2013).

[15] A. Mantile. Quantum evolution in the regime of quantum wells in a semiclassical island with artificial interface conditions. J. Math. Phys., 55, P. 092104 (2014).

[16] G. Nenciu, Linear adiabatic theory. Exponential estimates. Comm. Math.Phys., 152(3),P. 479-496 (1993).

[17] C. Presilla, J. Sjöstrand. Transport properties in resonant tunneling heterostructures. J. Math. Phys., 37(10), P. 4816-4844 (1996).

[18] C. Presilla, J. Sjöstrand. Nonlinear resonant tunneling in systems coupled to quantum reservoirs. Phys. Rev. B: Condensed matter, 55(15), P. 9310-9313 (1997).

[19] B. Simon, The definition of molecular resonance curves by the method of exterior complex scaling, Phys. Lett., 71A, P. 211-214 (1979).

[20] E. Skibsted. On the evolution of resonance states. J. Math. Anal. Appl., 141(1),P. 27-48 (1989).

[21] J. Sjöstrand, M. Zworski. Complex scaling and the distribution of scattering poles. J. Amer. Math. Soc., 4, P. 729-769 (1991).

[22] D.R. Yafaev. Mathematical Scattering Theory: Analytic theory. Mathematical Surveys and Monographs vol. 158, American Mathematical Society, Providence (2010). 